\title{
Primary pulmonary artery sarcoma with intrapulmonary metastases based on PET/CT imaging: a case report and literature review
}

\author{
Bo Pan ${ }^{1}$, Shi-Cun Wang ${ }^{1}$, Zong-Ke Chen ${ }^{2}$, Xi Chen ${ }^{3}$ \\ ${ }^{1}$ Department of Nuclear Medicine, The First Affiliated Hospital of USTC, Division of Life Sciences and Medicine, University of Science and \\ Technology of China, Hefei, China; ${ }^{2}$ Department of Pathology, The First Affiliated Hospital of USTC, Division of Life Sciences and Medicine, \\ University of Science and Technology of China, Hefei, China; ${ }^{3}$ Department of Orthopaedics, The First Affiliated Hospital of USTC, Division of \\ Life Sciences and Medicine, University of Science and Technology of China, Hefei, China \\ Correspondence to: Xi Chen. Department of Orthopaedics, First Affiliated Hospital, University of Science and Technology of China, No.1 Swan Lake \\ Road, Shushan District, Hefei 230036, China. Email: chenxsky@126.com.
}

\begin{abstract}
Primary pulmonary artery sarcoma is an extremely rare and highly aggressive malignant tumor of cardiovascular system. It is usually misdiagnosed as pulmonary thromboembolism due to its atypical clinical features and similar clinical symptoms. Different from published reports, our case received both enhanced CT and ${ }^{18}$ F-FDG PET/CT examination before the pathologic result, and lung metastases had already occurred at the time of diagnosis. We herein reported a case of 41-year-old female patient who suffered from cough and chest tightness for more than a month. Laboratory examination indicated that both blood routine and tumor markers were within the normal range, and only the D-dimer slightly elevated. Contrast-enhanced chest computed tomography showed right pulmonary artery lesion and multiple nodular located right upper lung, the lesion was mild heterogeneous enhancement. No obvious abnormalities were found in deep vein of bilateral lower extremities on ultrasonography. In order to confirm the nature of these lesions, PET/CT scan was performed, which revealed stripe hypermetabolism in right pulmonary artery and nodular hypermetabolism in right upper lung, and the rest of the whole-body PET imaging were negative, a diagnosis of primary pulmonary artery malignancy with pulmonary metastases was made, and pulmonary thromboembolism was ruled out. Biopsy of right pulmonary lesions was performed and histopathological examination indicated pulmonary artery sarcoma. She only received palliative conservative medical treatment because the disease was late stage according to the tumor-node-metastasis (TNM) staging system, and did not acceptable surgical treatment, and was in good health during recent follow-up. Our study suggested that 18F-FDG PET/CT image is a good approach for the diagnosis of pulmonary artery sarcoma and could provide adjunct value for further treatment.
\end{abstract}

Keywords: Pulmonary artery sarcoma; 18F-FDG; PET/CT; intrapulmonary metastases; case report

Submitted Mar 07, 2020. Accepted for publication Oct 22, 2020.

doi: 10.21037/apm-20-630

View this article at: http://dx.doi.org/10.21037/apm-20-630

\section{Introduction}

Sarcoma is a malignant tumor derived from mesenchymal tissue. Pulmonary artery sarcoma (PAS) is a rare site of sarcoma and fewer than 400 cases have been reported in the literatures, which only accounts for approximately $0.001-0.003 \%$ of all sarcomas $(1,2)$. Consequently, PET/
CT imaging used for PAS is even rarer, only a few case reports have described these lesions, but all these cases were misdiagnosed as pulmonary embolism or pneumonia at initial diagnosis with the imaging manifestations of FDG high uptake on PET/CT, and finally confirmed as PSA by postoperative histopathological results (3-5). Herein we 


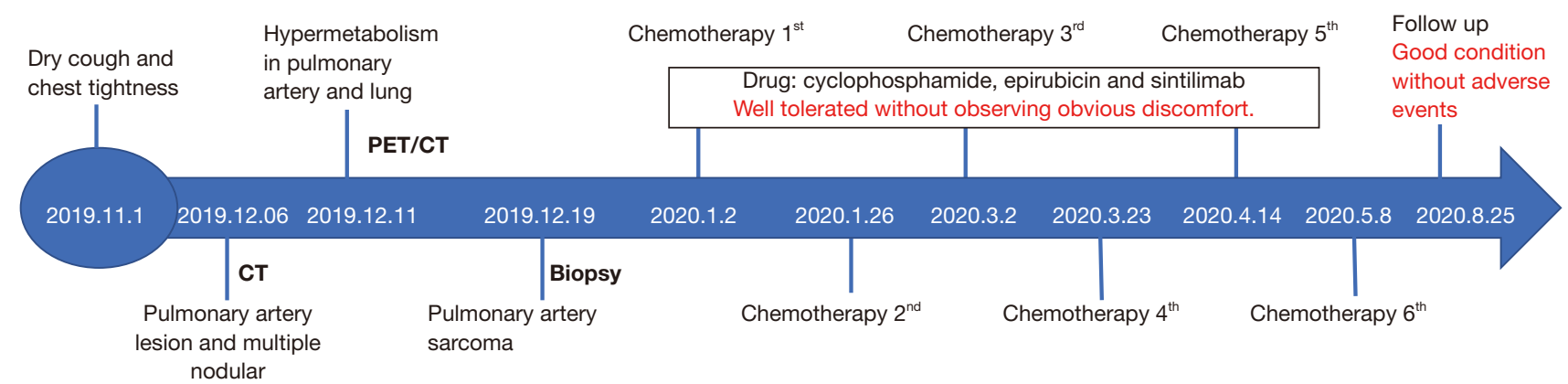

Figure 1 Time of clinical history for pulmonary artery sarcoma. PET indicates positron emission tomography; CT indicates compute tomography.

present a case of 41-year-old man with initial diagnosis of PAS. She underwent a biopsy of the right lung and histopathology examination revealed pulmonary artery spindle cell sarcoma, she did not undergo operation because the disease is late stage, and received chemotherapy and immunotherapy. This rare case showed hypermetabolic within the right pulmonary artery accompanying multiple metastases in the right upper lung on PET/CT, indicating malignant lesions. The time-line of the clinical history for this patient was shown in Figure 1.

We present the following article in accordance with the CARE reporting checklist (available at http://dx.doi. org/10.21037/apm-20-630).

\section{Case presentation}

The study was approved by committee ethics board of the first affiliated hospital of university of science and technology of china (No. 2018-ky028). All procedures performed in studies involving human participants were in accordance with the ethical standards of the institutional and/or national research committee(s) and with the Helsinki Declaration (as revised in 2013). Written informed consent was obtained from the patient.

A 41-year-old women presented with dry cough and chest tightness for more than a month. Plain CT scan of the chest at a local hospital revealed a soft mass in the right hilar and multiple nodules in right upper lung, and she was referred to our hospital for further treatment. Contrast enhanced CT indicated pulmonary artery filling defect with multiple nodular lesions in the right lung, color doppler echocardiography showed moderate pulmonary hypertension and decreased left ventricular diastolic function, and no abnormalities were seen in deep venous of both lower extremities with sonography, a diagnosis of pulmonary artery sarcoma with right lung metastatic tumors was made. No enlargement of liver, spleen, and superficial lymph nodes were detected. Her initial blood pressure was $108 / 70 \mathrm{mmHg}$, and heart rate was 89 beats per minute, she took approximately 20 breaths per minute, and the $\mathrm{CO}_{2}$ binding capacity was $23.20 \mathrm{mmol} / \mathrm{L}$ (normal range: 20.00-30.00). laboratory tests showed neuron-specific enolase slightly elevated to $23.09 \mathrm{ng} / \mathrm{mL}$ (reference range: $0.00-17.00 \mathrm{ng} / \mathrm{mL}$ ), the $\mathrm{D}$-dimer slightly elevated to $1.28 \mu \mathrm{g} / \mathrm{mL}$ (reference rang: $0.01-0.55 \mu \mathrm{g} / \mathrm{mL}$ ), tumor marker AFP, CEA and CA19-9 were all within normal limits, blood routine results were also in normal range. She had a history of cesarean section operation two month ago, and denied any history of infectious diseases such as hepatitis, tuberculosis, and malaria, and had no history of hypertension, heart disease, cerebrovascular disease, or psychosis, and no history of family tumor or genetic disease. To further assess the lesions of within right pulmonary artery and right lung, PET/CT scan was then performed. The lesion within right main pulmonary artery and nodules in right upper lung manifested increased FDG uptake [maximum standardized uptake value (SUVmax) 12.7, Figure 2], a pulmonary artery sarcoma with intrapulmonary metastasis were diagnosed, meanwhile, she was also diagnosed with pulmonary hypertension. Then right lung nodule biopsy was performed under CT-guided, histopathology examination showed malignant tumor mainly composed of spindle cells, and immunohistochemical staining was positive for Vim, TTF-1, CD34, and Bcl-2, and negative for CK7, Syn, CgA, and CD10, and the Ki-67 level was approximately $80 \%$, which suggested spindle cell sarcoma (Figure 3).

The clinical stage of the tumor was T4N0M0 and the KPS score was 80 . After a multidisciplinary consultation, she received conservative comprehensive treatment 
A
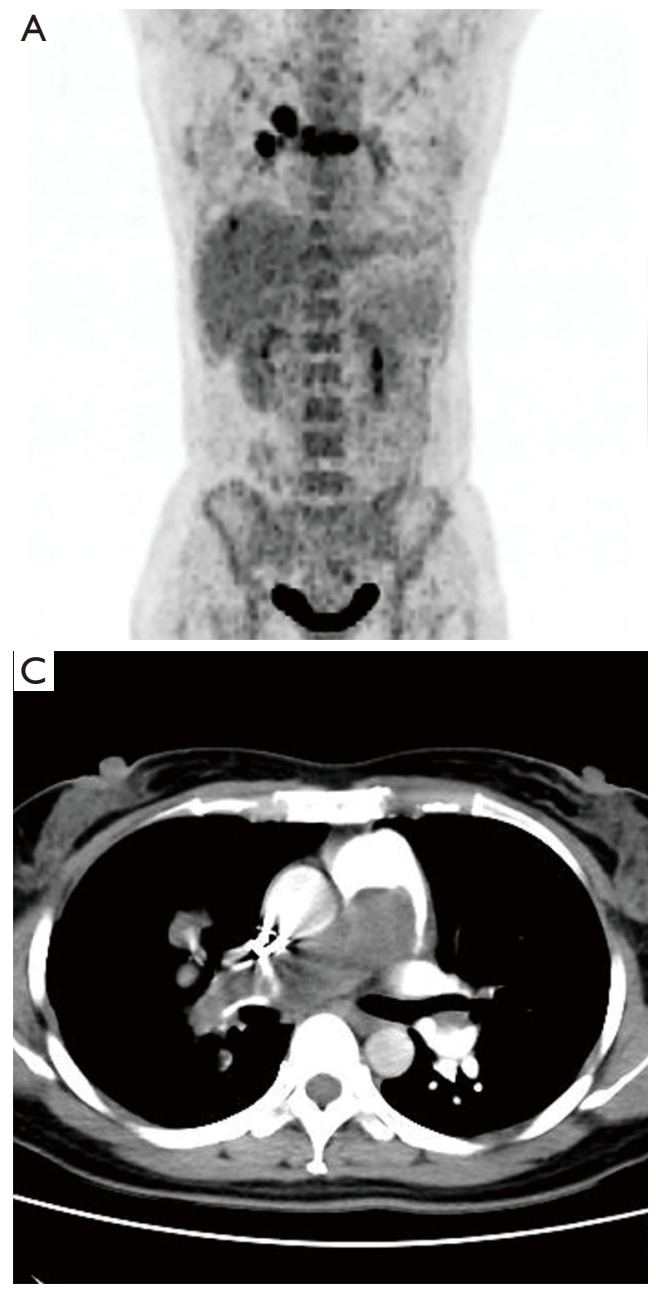

B
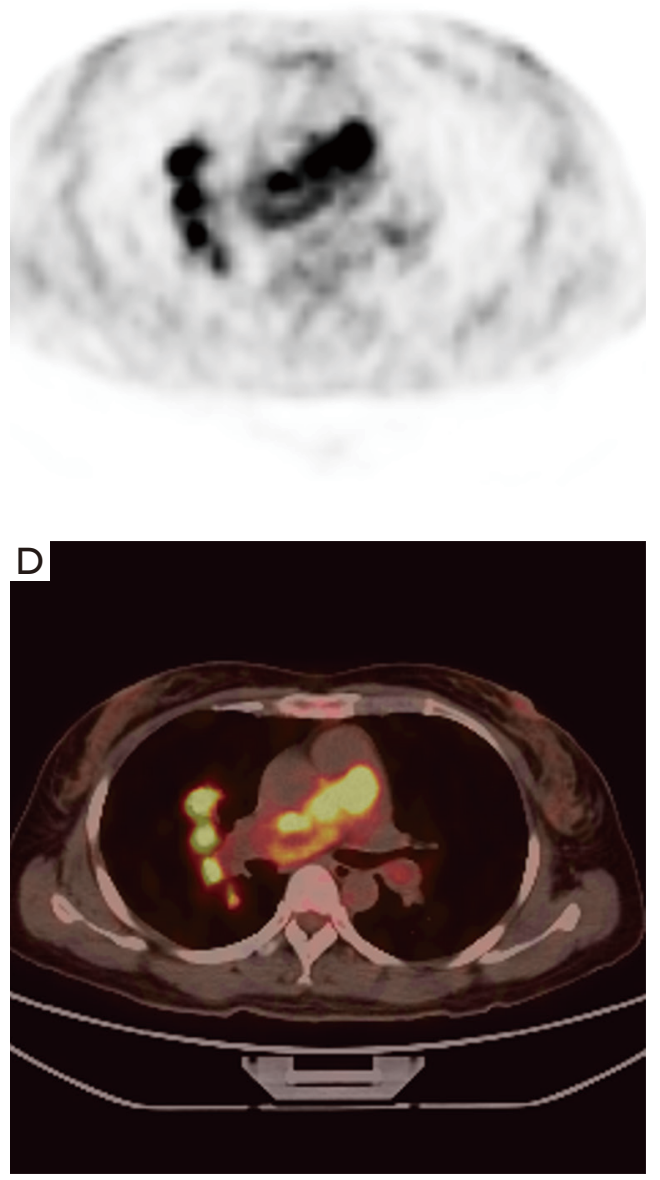

Figure 2 Radiographic examinations using CT and PET/CT. (A) Nodular hypermetabolism lesions were seen in mediastinum and superior lobe of right lung on PET image; (B) a striped hypermetabolic focus in the main right pulmonary artery and multiple hypermetabolic foci in superior lobe of right lung were observed on transverse PET image; (C) filling defects were seen in right main and the branches of pulmonary artery on axial contrast enhanced CT; (D) multiple hypermetabolic foci in right upper lung and striped FDG avid in the main right pulmonary artery were seen on axial fusion image. PET, indicates positron emission tomography; CT, indicates compute tomography; FDG, fluoro-deoxy-glucose.

instead of surgery or radiotherapy. And she was willing to receive chemotherapy plus immunotherapy. she received chemotherapy with cyclophosphamide $1.2 \mathrm{~g}$ per day and epirubicin $80 \mathrm{mg}$ per day for 5 courses of chemotherapy. At the same time, she received immunotherapy with sintilimab $100 \mathrm{mg}$ per day 5 courses of chemotherapy, and is in the hospital for six-cycle chemotherapy, no obvious adverse reactions appeared during chemotherapy. She was in good health during 6-month of voluntary follow-up with color doppler echocardiography, and nothing adverse or unexpected happened. She agreed to have her clinical data and images published in journals for use in medical academic exchanges.

She underwent a total of 6 cycles of chemotherapy so far, and his symptoms were significantly improved compared with those before treatment. Symptoms of chest distress were mild, shortness of breath was significantly improved, and he had no other complaints of discomfort.

\section{Discussion}

Primary pulmonary artery sarcoma is a rare malignant 


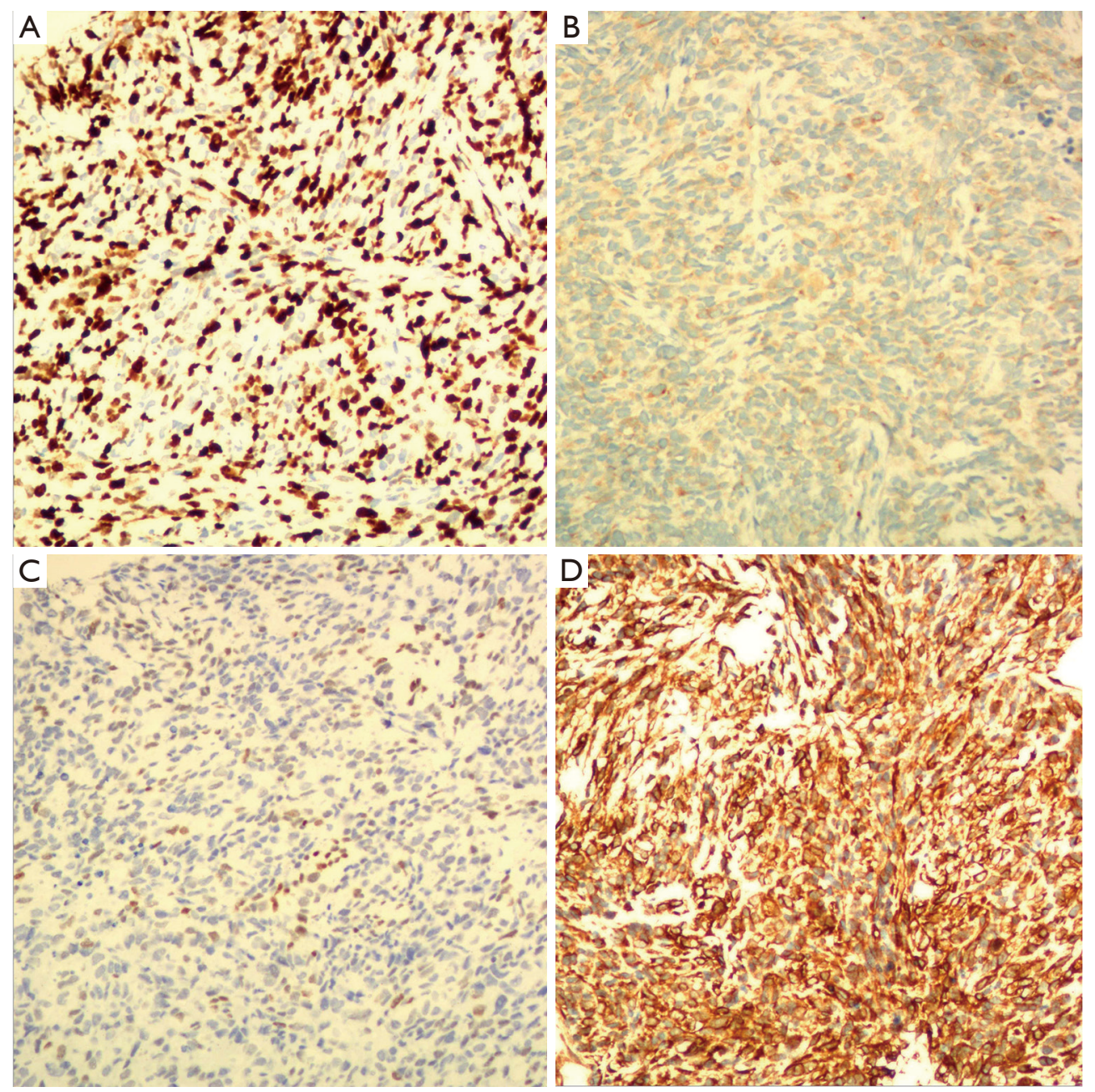

Figure 3 Histopathological analysis. (A) IHC staining was positive for Vim; (B) IHC was positive for TTF-1 (weak); (C) IHC was positive for Bcl-2; (D) the Ki-67 proliferation index was approximately $80 \%$. All magnifications were $\times 200$. IHC, immunohistochemistry.

tumor originating from the intima or subintima of the pulmonary artery, less than 400 cases have been reported since it was first described in 1923 by Mandelstamm $(6,7)$. The exact etiology is unclear, and the incidence is about $0.001 \%$ to $0.003 \%$ (2), the disease is more occult at early stage, the non-specific symptoms include chest pain, cough, dyspnea, hemoptysis or sputum with blood, syncope and asymptomatic. Because clinical manifestations of pulmonary artery sarcoma lack specific symptoms and it is difficult to make accurate diagnosis at early stage, most of the patients were misdiagnosed as pulmonary thromboembolic disease before surgery (8-10). After repeated thrombolytic therapy failed, and the diagnosis of pulmonary artery sarcoma was made during surgical treatment. The imaging findings of echocardiography, and pulmonary vascular magnetic resonance imaging were similar to those of pulmonary embolism, the 'wall eclipsing sign' on pulmonary artery computed tomography angiography is typical characteristic for diagnosis of PAS (1). Pulmonary artery sarcoma could manifest increased FDG uptake on PET/CT imaging, however, pulmonary artery thromboembolism usually shows radioactive defect, which is important for the differential diagnosis between pulmonary artery sarcoma and pulmonary thromboembolism, and ${ }^{18} \mathrm{~F}-\mathrm{FDG}$ PET/ CT have been shown useful in the diagnosis of pulmonary artery sarcoma and also could contribute to an accurate diagnosis for determining pulmonary artery malignancy $(4,11,12)$. In addition, ${ }^{18}$ F-FDG PET/CT could provide whole body condition through once examination, and whether the tumor has distant metastasis. Accordingly, 
it could provide useful information for tumor diagnosis and staging. However, not all pulmonary artery sarcomas show hypermetabolic on ${ }^{18} \mathrm{~F}$-FDG PET/CT imaging, and the minority of pulmonary artery intimal sarcoma can present hypometabolism at ${ }^{18} \mathrm{~F}$-FDG PET/CT image, which is easily misdiagnosed as pulmonary embolism (8). The main imaging features of pulmonary artery sarcoma include filling defects with hypermetabolism on PET/CT images, expansion the artery, metastasis to the lungs or mediastinum, and delayed enhancement on CT image, which are different from pulmonary artery blood thrombosis. Although there was no standard TNM staging system at present, optimal treatment regimens are adopted for different staging tumor. Pulmonary artery sarcoma is a rare and highly lethal disease, and its diagnosis and optimal treatment strategy are not conclusive. Surgery is preferred treatment choice for pulmonary sarcoma up to now. Patients will benefit from early diagnosis and complete surgical resection, and postoperative adjuvant treatment of management may prolong the patient's survival (13). Patients with untreated pulmonary artery sarcoma would be a median survival of 6 weeks (14). Although the surgery and the newer upcoming treatment strategies may prolong the median survival of patients, the long-term prognosis remains extremely poor (15).

In our case study, a complete auxiliary examination was performed with pulmonary artery angiography and ${ }^{18} \mathrm{~F}$-FDG PET/CT imaging for our case, pulmonary angiography suggested mild enhancement of pulmonary artery lumen lesions, and pulmonary embolism was first considered. ${ }^{18} \mathrm{~F}$-FDG PET findings indicated that pulmonary artery lesion accompanied by multiple nodules in the right upper lung, a pulmonary artery malignancy accompanied by right metastasis was made, and pathologic findings of right lung nodule revealed sarcoma. The disease was at an advanced stage, so surgical treatment could not be performed, Chemotherapy plus immunotherapy is first-line treatment for pulmonary artery sarcoma, to our knowledge, sintilimab used as a treatment for PAS has not been reported previously. Up to now, the patient is in good condition and is under follow-up. The limitations of our study are that we cannot predict eventual survival while patients are still being treated and followed up. Chemotherapy combined with immunotherapy may be a good treatment approach for advanced pulmonary artery sarcoma, and patient would benefit from it. When a lesion in the pulmonary artery cavity demonstrates hypometabolism on PET imaging, it is easily to rule out pulmonary embolism due to its hypometabolism on PET image. The case presented with pulmonary arterial luminal mass, enhanced CT indicated mild enhancement of the lesion, and the size of the lesion did not change after anticoagulant treatment. The lesions in the arterial luminal indicated hypermetabolism accompanied by hypermetabolism nodules in the right lung on ${ }^{18} \mathrm{~F}-\mathrm{FDG}$ PET/CT imaging. Pulmonary artery malignancy with intrapulmonary metastasis was first considered, and pulmonary thromboembolism could be excluded. Therefore, when there were nodular or striped hypermetabolic foci in the pulmonary artery cavity at ${ }^{18}$ F-FDG PET imaging, primary pulmonary artery sarcoma should be taken into consideration firstly.

\section{Conclusions}

Primary pulmonary artery sarcoma should be firstly taken into consideration when a nodular or striped hypermetabolism of intraluminal lesions of pulmonary artery on PET/CT image. Other findings include metastasis to lung or mediastinum may provide adjunct diagnostic value for pulmonary artery sarcoma.

\section{Acknowledgments}

Thanks to Dr. Zhao Jieting for her help in improving the case data.

Funding: This work was supported by the Strategic Priority Research Program of the Chinese Academy of Sciences (No. XDB39000000).

\section{Footnote}

Reporting Checklist: The authors have completed the CARE reporting checklist. Available at http://dx.doi.org/10.21037/ apm-20-630

Peer Review File: Available at http://dx.doi.org/10.21037/ apm-20-630

Conflicts of Interest: All authors have completed the ICMJE uniform disclosure form (available at http://dx.doi. org/10.21037/apm-20-630). The authors have no conflicts of interest to declare.

Etbical Statement: The authors are accountable for all aspects of the work in ensuring that questions related to the accuracy or integrity of any part of the work are 
appropriately investigated and resolved. The study was approved by committee ethics board of the first affiliated hospital of university of science and technology of china (NO.2018-ky028). All procedures performed in studies involving human participants were in accordance with the ethical standards of the institutional and/or national research committee(s) and with the Helsinki Declaration (as revised in 2013). Written informed consent was obtained from the patient.

Open Access Statement: This is an Open Access article distributed in accordance with the Creative Commons Attribution-NonCommercial-NoDerivs 4.0 International License (CC BY-NC-ND 4.0), which permits the noncommercial replication and distribution of the article with the strict proviso that no changes or edits are made and the original work is properly cited (including links to both the formal publication through the relevant DOI and the license). See: https://creativecommons.org/licenses/by-nc-nd/4.0/.

\section{References}

1. Gan HL, Zhang JQ, Huang XY, et al. The wall eclipsing sign on pulmonary artery computed tomography angiography is pathognomonic for pulmonary artery sarcoma. PLoS One 2013;8:e83200.

2. Li B, Zhang Y, Cai L, et al. Primary pulmonary artery sarcoma differentiated from pulmonary thromboembolism by ventilation-perfusion scan. Long survival of the patient. Hell J Nucl Med 2015;18:166-8.

3. Feng Y, Xu T, Wang C, et al. Pulmonary Artery Sarcoma Mimicking Pneumonia: Case Report and Literature Review. Int J Clin Exp Pathol 2019;12:3620-4.

4. Wu Y, Huang J, Wang Q, et al. Whole-exome sequencing insights into pulmonary artery sarcoma mimicking pulmonary embolism: a case report and review. Onco Targets Ther 2019;12:6227-35.

5. Xi XY, Gao W, Gong GN, et al. Value of 18F-FDG $\mathrm{PET} / \mathrm{CT}$ in differentiating malignancy of pulmonary

Cite this article as: Pan B, Wang SC, Chen ZK, Chen X. Primary pulmonary artery sarcoma with intrapulmonary metastases based on PET/CT imaging: a case report and literature review. Ann Palliat Med 2021;10(6):7013-7018. doi: 10.21037/ apm-20-630 artery from pulmonary thromboembolism: a cohort study and literature review. Int J Cardiovasc Imaging 2019;35:1395-403.

6. Mandelstamm M. Uber primare neubildungen des herzens. Virchows Arch 1923;245:43-54.

7. Mussot S, Ghigna MR, Mercier O, et al. Retrospective institutional study of 31 patients treated for pulmonary artery sarcoma. Eur J Cardiothorac Surg 2013;43:787-93.

8. Liu X, Hou J, Wang XD, et al. An intimal sarcoma of pulmonary artery mimicking pulmonary embolism: a case report and literature review. Respirol Case Rep 2017;5:e0248.

9. Panfeng X, Zheying Z, Jie W, et al. Successful Diagnosis of Pulmonary Artery Sarcoma by Contrast-Enhanced Computed Tomography. J Thorac Oncol 2008;3:772-3.

10. Lee Y, Kim HJ, Yoon H, et al. Clinical Characteristics and Treatment Outcomes of Primary Pulmonary Artery Sarcoma in Korea. J Korean Med Sci 2016;31:1755-60.

11. Kamaleshwaran KK, Pattabiraman V, Mehta S, et al. Spindle cell sarcoma of pulmonary artery mimicking thromboembolism with lung metastasis detected in fluorine-18 fluorodeoxyglucose positron emission tomography/computed tomography. Indian J Nucl Med 2014;29:249-51.

12. Kim C, Kim MY, Kang JW, et al. Pulmonary Artery Intimal Sarcoma versus Pulmonary Artery Thromboembolism: CT and Clinical Findings. Korean J Radiol 2018;19:792-802.

13. Bandyopadhyay D, Panchabhai TS, Bajaj NS, et al. Primary Pulmonary Artery Sarcoma: A Close Associate of Pulmonary embolism-20-year Observational Analysis. J Thorac Dis 2016;8:2592-601.

14. Krüger I, Borowski A, Horst M, et al. Symptoms, diagnosis, and therapy of primary sarcomas of the pulmonary artery. Thorac Cardiovasc Surg 1990;38:91-5.

15. Chang DY, Lin KC, Pan JY, et al. Pulmonary artery intimal sarcoma: a case report and literature review. Respirology Case Reports 2020;8:e00530. 